\title{
Uterine fibroid treatment with magnetic resonance-guided focused ultrasound surgery (MRgFUS): Inclusion and exclusion criteria
}

Sara Mascaretti", Fabiana Ferrari, Anna Miccoli, Francesco Arrigoni, Carlo Masciocchi and Giulio Mascaretti

*Correspondence: saramascaretti@gmail.com

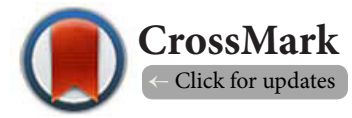

Department of Biotechnological and Applied Clinical Sciences, University of L'Aquila, Italy.

\begin{abstract}
Background: Patients affected by uterine fibroids were evaluated to define inclusion and exclusion criteria for treatment with Magnetic Resonance-guided Focus Ultrasound Surgery (MRgFUS), the non-invasive technique considered as an efficient alternative to hysterectomy and myomectomy.

Methods: From October 2011 to December 2015, 174 patients aged from 23 to 51 years, affected by uterine fibroids (size ranging from 2 to $14 \mathrm{~cm}$ ) were evaluated in our clinical unit, to define candidature for MRgFUS treatment. Symptomatic patients were submitted to preliminary MRI. The severity of symptoms was evaluated with the UFS-QOL questionnaire. All patients were clinically examined to verify the absence of all conditions reported by the exclusion criteria grid.

Results: 54 out of 174 patients were considered suitable for MRgFUS and treated; in 16 out of 174 patients, suitable for MRgFUS, treatment was declined because MRI evaluation, performed on the treatment's day, showed interposition of bowel and/or bladder. 104 out of 174 were not candidate to MRgFUS, due to interposition of bowel and bladder (46/104), presence of pedunculated fibroids (18/104), large abdominal scars (18/104), close proximity to the spine and nerve roots (22/104).

Conclusions: In our study, applying the inclusion and exclusion criteria, the percentage of treatable and treated patients was $40 \%$ and $31 \%$, respectively. Diriment in the choice of MRgFUS over myomectomy is the possibility to achieve the same result (disappearance or marked reduction of the fibroid) without risks and complications related to conventional surgery. Furthermore, hospitalization times are sensibly reduced to one day only and the convalescence is maximum 2-3 days.
\end{abstract}

Keywords: MRgFUS, ultrasound surgery, uterine fibroids, inclusion and exclusion criteria

\section{Introduction}

Fibroids are benign tumours growing in the uterus. They are symptomatic in up to $25 \%$ of women in childbearing age [1]. Symptoms can include heavy and prolonged menstrual bleeding, pain or pressure in the pelvic area, pollakiuria and difficulty in emptying the bladder, constipation and abdominal bloating [2]. Hysterectomy is the most common treatment for uterine fibroids [3], but it implies long hospitalization, post-operative complications and loss of the organ [4]. All these implications have led during the years to an increasing demand for noninvasive resolving techniques. Different procedures can be employed: Myomectomy, uterine artery embolization (UAE), short-term treatment with GnRH agonists [5] and MR-guided high intensity focused ultrasound surgery (MRgFUS). The employment of methods allowing conservation of the organ without surgical cuts has several advantages over open surgery procedures: [6-8] Lower morbidity, and shorter recovery and hospitalization times. Absence of surgical cuts, sedation instead of general anaesthesia, considerably reduced recovery times and a definitely better quality of life for the patient are all advantages offered by MRgFUS. In fact, patients submitted to MRgFUS usually go back to normal within 48 hours. About 10 days are usually needed after UAE and 6 weeks after myomectomy or hysterectomy [9]. Additionally, multicentre studies on fertility are recruiting women with symptomatic uterine fibroids who desire pregnancy [10-12]. The non-invasive nature 
of MRgFUS, through which it is possible to thermoablate uterine fibroids without damaging the surrounding healthy tissues, suggests that MRgFUS is a safe approach for women who want to preserve their fertility. The aim of this study was to evaluate the inclusion and exclusion criteria for MRgFUS treatment, in order to avoid side effects, using a conservative therapy in women in childbearing age, with the possibility of saving the uterus without fibrotic scarring. The correct analysis of the exclusion criteria listed on the specific grid ensures higher success percentages. The accurate selection of patients before the treatment proves to be essential for an excellent result, as much as the treatment itself.

\section{Methods}

From October 2011 to December 2015, 174 patients aged from 23 to 51 (mean, 37 years) and affected by uterine fibroids (sized from 2 to $14 \mathrm{~cm}$ ) were treated at the Department of Radiology of the San Salvatore Hospital.

Patients were initially examined by a gynaecologist for clinical evaluation of symptoms and their possibility to participate in the MRgFUS trial. The gynaecological evaluation consists in anamnesis of the patient, assessment of symptom severity, verification of the presence of abdominal scars, and performance of a medical ultrasound examination.

Patients resulting positive to the gynaecological tests underwent MRI with and without contrast agents for the complete topographical and morphological evaluation of pelvis and fibroids. MRI selection criteria follows [13]. MRI offers information about site and number of fibroids, as well as possible involvement of abdominal organs.

After an accurate clinical evaluation, the patients were asked to fill in a questionnaire (the UFS-QOL) consisting of 8 questions regarding the SSS (Severity Symptoms Score), which was assessed using a Likert scale, with 5 points assigned to each question [14]. The resulting final score ranged from 8 to 40. The patients were asked to fill in an additional questionnaire on their quality of life in terms of procreation, mood, dynamic temperament, control, self- and sexual awareness.

The present study excluded patients who presented standard contraindications to MRI, such as presence of an implanted device, even if non-metallic, obesity (weight $>110$ $\mathrm{kg}$ ), difficulty in tolerating the prolonged stationary prone position in the MR scanner during the treatment, hypersensitivity to the contrast agent or claustrophobia. Patients who were not able to understand the instructions or to communicate their sensations during the treatment were also excluded. An additional exclusion parameter was the presence of unfavourable clinical conditions such as unstable cardiovascular pathologies, cerebrovascular diseases, haemolytic anaemia, anti-coagulation therapy or haemostasis disorders. Other exclusion causes were the concurrence of uterine pathologies such as malignant tumour and pelvic inflammatory diseases (endometriosis). All patients were submitted to a preliminary MRI exam of the pelvis, necessary also for the correct visualiza- tion of distance between uterus and skin and between uterus and spine, possible interposition of intestines, and relation between bladder and uterus. The patients were considered technically treatable with MRgFUS when the fibroids could be achieved by the ultrasound beam. The distance of the fibroids from the skin was also taken into consideration, and a maximum limit of $14 \mathrm{~cm}$ was established. Patients with an intestinal interposition on the ultrasound beam path were also excluded to avoid damages to the intestinal wall, that can absorb the ultrasound energy and be perforated. This condition can only be observed during the procedure, and is almost impossible to predict.

Further exclusion criteria are the following:

- Pedunculated fibroids that could detach inside the peritoneal cavity after the treatment

- Calcified fibroids hindering penetration of the beam

- Fibroids showing at MR a vascularised structure and particularly irregular borders, inadequate to reach the required therapeutic temperatures

- Patients with important abdominal scarring which can divert and absorb energy, causing pain and burns.

Patients were also submitted to clinical and instrumental follow-up studies after six months and one year.

There are some differences in the eligibility criteria between our study and the American study by Millie A. Behera et al., [15] Notably, the North Carolina study presents elements such as desired future fertility, adenomyosis [16], large or multiple fibroids, intracavitary fibroids as exclusion criteria, that in our study are considered as inclusion criteria.

\section{Results}

After all the preliminary examination steps, the following results were obtained. The number of patients who were considered unsuitable for the MRgFUS is 104 out of 174 , because of the interposition of bowel and/or bladder (46/104), presence of pedunculated fibroids (18/104), large abdominal scars (18/104), close proximity to the spine and nerve roots (22/104). Considering the total percentage (100\%) of examined patients, $60 \%$ was excluded for the following reasons: interposition of the bowel and the bladder (26\%), pedunculated fibroids $(10 \%)$, large abdominal scar (10\%), close proximity to spine and sacrum (13\%).

The number of patients who were considered suitable for the MRgFUS treatment and effectively treated is 54 out of 174 . In 16 out of 174 patients, suitable for MRgFUS, treatment was declined because MRI evaluation, performed on the day of treatment, showed interposition of the bowel and/ or bladder, which were located anteriorly to the uterine wall. This is not reported as an exclusion criteria, because it is an unpredictable and adjustable medical case.

Considering the total percentage (100\%) of examined patients, $40 \%$ were included in the study. In $9 \%$ of the total the treatment was declined due to bowel interposition, observed on the same day of the treatment. The treatment was successfully 
Mascaretti et al., Gynecology 2016, http://www.hoajonline.com/journals/pdf/2052-6210-4-1.pdf

carried out in $31 \%$ of the patients (Tables 1 and 2 ).

Table 1. Eligible and non eligible patients.

\begin{tabular}{lll}
\hline Eligible patients & Number patients & $\%$ \\
\hline Obtained & 54 & 31 \\
Not obtained & 16 & 9 \\
\hline Not Eligible patients & Number patients & $\%$ \\
\hline Non acoustic window & 46 & 26 \\
Peduncolated fibroids & 18 & 10 \\
Abdominal scar & 18 & 10 \\
Proximity coulumn & 22 & 13 \\
\hline
\end{tabular}

Table 2. Exclusion criteria.

\begin{tabular}{lll}
\hline Exclusion Criteria & Number Patients & $\%$ \\
\hline Non acustic window & 46 & 26 \\
Peduncolate fibroids & 18 & 10 \\
Abdominal scar & 18 & 10 \\
Proximity to vertebral coulumn & 22 & 13 \\
\hline
\end{tabular}

\section{Discussion}

Surgery using Magnetic Resonance-guided Focused Ultrasound (MRgFUS) is a non invasive day-surgery procedure that employs high quantities of ultrasound waves (HIFU) to destroy the uterine fibroids without any incidence on the other tissues around the affected area. The procedure is carried out under Magnetic Resonance guidance that helps the operator to observe, guide and manipulate the treatment with continuity. The system reproduces the phenomenon of a hand lens that makes the solar energy converge on one point creating a flame to burn a leaf. The energy of the focused ultrasounds is directed toward a small portion of the fibroid, increasing its temperature just enough to originate the thermal ablation (destruction of the cell), without any effect on the other tissues. The ultrasonic thermal impulses are repeated until the entire volume is treated.

In our medical unit, uterine fibroids are presently treated with the MRgFUS technique instead of the traditional surgical procedure. The temperature reached inside the tissue $\left(65-85^{\circ} \mathrm{C}\right)$ destroys it through a coagulative necrosis. The introduction of this therapeutical method has noticeably broadened the set of options available for patients affected by uterine fibroids. This treatment is a non-invasive option also for those patients affected by symptomatic fibroids. The majority of patients treated with the MRgFUS technique takes immediate advantage and enjoys symptomatic relief for more than two years. Only 10 to $15 \%$ of the patients require further treatments.

The exclusion criteria are: interposition of intestinal loops between the abdominal wall and the mass, which entails the absence of acoustic window ( $26 \%$ of examined patients underwent this criteria); close proximity of the fibroids to the spine, and thus to the nerve roots ( $13 \%$ of examined patients); possible presence of abdominal scars placed anteriorly to the mass ( $10 \%$ of examined patients).

In the previous literature on the issue [15], criteria such as desired fertility, vertical scars, tattoos, too much fibroid volume, too small fibroids, intracavitary fibroids, adenomyosis, have been considered as exclusion criteria. Thanks to our unit experience, the above mentioned criteria haven't been regarded as exclusion criteria because solutions have been found for some of them and we haven't encountered problems in treating patients responding to others of these criteria. Women desiring to preserve their fertility were included in our study because the MRgFUS treatment do not endanger it, on the contrary, it improves it. In order to find a solution in the presence of large abdominal scars, a heat absorption patch was used when possible. In this way, the sonications were performed without any cutaneous injury. None of the fibroids has been considered as "too large", since even a reduction of the mass is acknowledged as a positive result to restore the regular anatomy of the organ or to reduce symptoms. In the same way, even the samllest fibroid has been considered to worth a treatment. Women affected by intracavitary fibroids and adenomyosis have been regularly treated with MRgFUS since no contraindications were encountered before, during or after the treatment.

Other solutions were found to limits such as interposition of the bladder and of the intestinal loops, and in some specific case proximity of the spine, so that they weren't considered as exclusion criteria anymore, thus allowing more patients to be treated. A different positioning of the intestinal loops in the retro-uterine pouch, obtained using an inversion therapy table and a pelvic abdominal massage, was sometimes necessary, keeping the bladder well relaxed with a NMR control exam. Once the repositioning of the intestinal loops is confirmed, the bladder is emptied to guarantee that the anterior part of the uterus be completely in contact with the abdominal wall. In some cases, when uterus and fibroid were in proximity of the spine, a probe cover filled with aqua-sonic gel was interposed, upon rectal application, in order to create a vast sonic-safe zone between the lesion and the spine itself. This technique avoided all the complications on the nerve roots of the spine.

\section{Conclusion}

The critical application of the discussed inclusion and exclusion criteria allowed the following result to be reached. Forty per cent of the total examined patients were included in the study. In $9 \%$ of the total the treatment was declined due to bowel interposition, observed on the same day of the treatment. The treatment was successfully carried out in $31 \%$ of the patients.

\section{Competing interests}

The authors declare that they have no competing interests. 
Mascaretti et al., Gynecology 2016,

\section{Authors' contributions}

\begin{tabular}{|l|c|c|c|c|c|c|}
\hline Authors' contributions & SM & FF & AM & FA & CM & GM \\
\hline Research concept and design & -- & -- & -- & $\checkmark$ & -- & -- \\
\hline Collection and/or assembly of data & -- & -- & $\checkmark$ & -- & -- & -- \\
\hline Data analysis and interpretation & $\checkmark$ & -- & -- & -- & -- & -- \\
\hline Writing the article & $\checkmark$ & -- & -- & -- & -- & -- \\
\hline Critical revision of the article & -- & -- & -- & -- & $\checkmark$ & $\checkmark$ \\
\hline Final approval of article & $\checkmark$ & -- & -- & -- & $\checkmark$ & $\checkmark$ \\
\hline Statistical analysis & $\checkmark$ & $\checkmark$ & -- & -- & -- & -- \\
\hline
\end{tabular}

\section{Publication history}

Senior Editor: Kaei Nasu, Oita University, Japan.

Editor: Seung-Yub Ku, Seoul National University, Korea.

Received: 05-Apr-2016 Final Revised: 07-Jun-2016

Accepted: 15-Jun-2016 Published: 24 -Jun-2016

\section{References}

1. Ryan GL, Syrop CH and Van Voorhis BJ. Role, epidemiology, and natural history of benign uterine mass lesions. Clin Obstet Gynecol. 2005; 48:312-24. | Article | PubMed

2. Drayer SM and Catherino WH. Prevalence, morbidity, and current medical management of uterine leiomyomas. Int I Gynaecol Obstet. 2015; 131:117-22. | Article | PubMed

3. Vilos GA, Allaire C, Laberge PY, Leyland N, Vilos AG, Murji A and Chen I. The management of uterine leiomyomas. J Obstet Gynaecol Can. 2015; 37:157-81. | Article | PubMed

4. Lalinec-Michaud $M$ and Engelsmann F. Anxiety, fears and depression related to hysterectomy. Can J Psychiatry. 1985; 30:44-7. | Article | PubMed

5. Puchar A, Feyeux C, Luton D and Koskas M. Therapeutic management of uterine fibroid tumors. Minerva Ginecol. 2015. | Article | PubMed

6. Ksiezakowska-Lakoma K, Zyla M and Wilczynski J. Removal of uterine fibroids by mini-laparotomy technique in women who wish to preserve their uterus and fertility. Wideochir Inne Tech Maloinwazyjne. 2016; 10:561-6. | Article | PubMed Abstract | PubMed FullText

7. van der Meij $E$ and Emanuel $\mathrm{MH}$. Hysterectomy for heavy menstrual bleeding. Womens Health (Lond Engl). 2016; 12:63-9. | Article | PubMed

8. Driessen SR, Sandberg EM, van den Haak L and Jansen FW. [Increase in laparoscopic hysterectomy: shift in indications and dilemmas]. Ned Tijdschr Geneeskd. 2015; 159:A9324. | Article I PubMed

9. Toor SS, Tan KT, Simons ME, Rajan DK, Beecroft JR, Hayeems E and Sniderman KW. Clinical failure after uterine artery embolization: evaluation of patient and MR imaging characteristics. J Vasc Interv Radiol. 2008; 19:662-7. | Article | PubMed

10. Myomas and reproductive function. Fertil Steril. 2008; 90:S125-30. | Article I PubMed

11. Zepiridis LI, Grimbizis GF and Tarlatzis BC. Infertility and uterine fibroids. Best Pract Res Clin Obstet Gynaecol. 2015. | Article I PubMed

12. Parazzini $F$, Tozzi $L$ and Bianchi S. Pregnancy outcome and uterine fibroids. Best Pract Res Clin Obstet Gynaecol. 2015. | Article | PubMed

13. Esclusione risonanza classica (RADIOLOGHE).

14. Spies JB, Coyne K, Guaou Guaou N, Boyle D, Skyrnarz-Murphy K and Gonzalves SM. The UFS-QOL, a new disease-specific symptom and health-related quality of life questionnaire for leiomyomata. Obstet Gynecol. 2002; 99:290-300. | Article | PubMed

15. Behera MA, Leong $M$, Johnson $L$ and Brown $H$. Eligibility and accessibility of magnetic resonance-guided focused ultrasound (MRgFUS) for the treatment of uterine leiomyomas. Fertil Steril. 2010; 94:1864-8. | Article I PubMed

16. Ferrari F, Arrigoni F, Miccoli A, Mascaretti S, Fascetti E, Mascaretti G, Barile A and Masciocchi C. Effectiveness of Magnetic Resonance-guided
Focused Ultrasound Surgery (MRgFUS) in the uterine adenomyosis treatment: technical approach and MRI evaluation. Radiol Med. 2016; 121:153-61. | Article | PubMed

\section{Citation:}

Mascaretti S, Ferrari F, Miccoli A, Arrigoni F, Masciocchi $\mathrm{C}$ and Mascaretti G. Uterine fibroid treatment with magnetic resonance-guided focused ultrasound surgery (MRgFUS): Inclusion and exclusion criteria. Gynecol. 2016; 4:1. http://dx.doi.org/10.7243/2052-6210-4-1 